\title{
Análisis del caso Solución numérica de ecuaciones diferenciales con incertidumbre y aplicaciones
}

\author{
Case analysis Numerical solution of differential equations with uncertainty \\ and applications
}

Azorín Penalva Ainhoa. ${ }^{1}$ \& Jorge Luis Yaulema Castañeda. ${ }^{2}$

\begin{abstract}
.
DOI: $\underline{\text { hhttps://doi.org/10.33262/concienciadigital.v4i3.1.1828 }}$

Introduction: For the solution of problems that need to be solved exactly. whose solution must be treated by means of numerical methods Objective: The present investigation aims to carry out a study of the numerical methods of Euler and Runge Kutta in order to make an approximation to the solution of random differential equations which use the calculus stochastic referring to the mean square. Methodology: Within the development process, Euler's methodology is analyzed in the first instance within the scalar case and later it is dimensioned to matrix problems, Results: obtaining an analysis of the application of numerical methods to the study of an electrical circuit which is develops with random noise, in the specific case of characteristic and irregular white noises they lead to other types of differential equations with a certain degree of uncertainty called stochastic differential equations. Conclusion: The Euler scheme allows us to conclude that the slow convergence and the restriction aspect of its region of absolute stability allows us to consider other methods where the convergence is greater, thus proposing an additional study of the Runge-Kutta random scheme, being a method superior to that of Euler for which its global order of convergence is fourth.
\end{abstract}

Keywords: Euler, Kutta Runge, numerical methods, differential equations

1 Escuela Superior Politécnica de Chimborazo, Ingeniería Eléctrica. Valencia, España aiazpe @epsa.upv.es, https://orcid.org/0000-0001-7265-1265

2 Escuela Superior Politécnica de Chimborazo, Facultad de Ciencias. Riobamba, Ecuador jorge.yaulema@espoch.edu.ec, https://orcid.org/0000-0002-0646-3984 


\section{Resumen.}

Introducción: Para la solución de problemas que requieren ser resueltos de manera exacta. cuya solución debe ser tratada por medio de los métodos numéricos Objetivo: La presente investigación tiene como objetivo realizar un estudio de los métodos numéricos de Euler y Runge Kutta con la finalidad de realizar una aproximación a la solución de ecuaciones diferenciales aleatorias las cuales utilizan el cálculo estocástico referente a la media cuadrática. Metodología: Dentro del proceso de desarrollo la metodología de Euler se analiza en primera instancia dentro del caso escalar y posteriormente se lo dimensiona a problemas matriciales, Resultados: obteniendo un análisis de la aplicación de los métodos numéricos al estudio de un circuito eléctrico el cual se desarrolla con ruido aleatorio, en el caso específico de ruidos blanco característicos e irregulares conducen a otro tipo de ecuaciones diferenciales con cierto grado de incertidumbre denominadas ecuaciones diferenciales estocásticas. Conclusión: El esquema Euler permite llegar a la conclusión de que la convergencia lenta y el aspecto de restricción de su región de estabilidad absoluta nos permite considerar otros métodos donde la convergencia es mayor, planteando así un estudio adicional del esquema aleatorio Runge- Kutta, siendo un método superior al de Euler por lo cual su orden de convergencia global es cuarto.

Palabras claves: Euler, Runge Kutta, métodos numéricos, ecuaciones diferenciales

\section{Introducción.}

Dentro de la aplicación de las matemáticas a situaciones del comportamiento diario y del mundo real nos encontramos a menudo con diferentes problemáticas, las cuales no pueden ser resueltas analíticamente o de manera exacta y cuya solución debe ser analizada mediante la resolución de problemas a través de los métodos numéricos (Azor et al. 2020).

Bossis, et al (2015), manifiesta que en cuanto a la planificación para la resolución de diferentes problemas como son el análisis de un circuito eléctrico, tomando en cuenta las medidas referentes a la realidad tenemos la necesidad de planificar modelos aleatorios, de tal manera que en la presente investigación se realizará la construcción numérica de soluciones para las ecuaciones diferenciales aleatorias a partir del esquema principal aleatorio de Euler mediante el cual se demostrará algunas condiciones para que la convergencia en media cuadrática sea estable, además se calculará el valor esperado y la varianza del proceso resultante de la aproximación (Seminario 2012)

\section{Metodologia}

La metodología utilizada en la presente investigación se basa en el análisis de métodos numéricos relacionados a la esquematización propuesta por Euler y Runge Kutta para la solución de ecuaciones diferenciales aleatorias de acuerdo al cálculo estocástico de la media cuadrática. 


\section{Método Numérico}

El método numérico se establece por medio de un procedimiento que se basa en las aproximaciones para encontrar la solución a ciertos problemas utilizando cálculos puramente aritméticos y operaciones lógicas elementales. Henrici (1962), el procedimiento que consiste en una lista finita de instrucciones que precisan una secuencia de operaciones algebraicas y lógicas que producen una aproximación de la solución a la problemática (Azor et al. 2020).

La eficiencia en el cálculo de dicha aproximación depende principalmente del escogimiento y facilidad de implementación del algoritmo y de las características especiales y limitaciones de los instrumentos de cálculo pudiendo generarse así un error de redondeo (Cortés, Jodar y Villafuerte, 2007).

\section{Esquema de Método Euler para ecuaciones diferencias aleatorias}

El esquema de Euler, presenta una convergencia lenta con una restricción de su región de estabilidad absoluta la cual considera otros métodos donde la convergencia puede ser mayor (Khodabin y Rostami, 2015 y Soong, 1973). Tal es el caso del esquema aleatorio de RugeKutta, que representa un método de orden superior que al de Euler, representado así por su orden de convergencia global cuarto (Azorín, 2020).

\section{Esquema del Método Runge Kutta para ecuaciones diferenciales aleatorias}

Los métodos numéricos son metodologías que parten de técnicas algebraicas y aritméticas utilizadas para poder resolver de forma aproximada de diferentes ecuaciones o sistemas de ecuaciones muy complejas. Las cuales analíticamente resultan muy dificultosos e incluso imposibles de obtener alguna solución (Villafuerte, 2007 y Aldana, et al 2016).

Puesto que, el proceso de convergencia lenta del método de Euler y lo restringido de su región de estabilidad absoluta, establecen la necesidad de métodos de orden de convergencia mayor (Oksendal, 1998).

Además, en cada paso, el método de Euler, se mueve a lo largo de la tangente de una cierta curva que esta "cerca" a la curva desconocida o buscada. Requiriendo por los mencionados motivos utilizar los métodos Runge-Kutta que extienden esta idea geométrica utilizando varias derivadas o tangentes intermedias en lugar de solo una, para aproximar la función desconocida (Lecca y Puente, 2006).

\section{Resultados}

Se muestran soluciones aproximadas de ciertas ecuaciones diferenciales con condiciones iniciales usando los métodos de Euler y Runge-Kutta aleatorios, tratando inicialmente la determinación del desplazamiento por un movimiento terrestre.

\subsection{Determinación del desplazamiento por un movimiento terrestre}

Para determinar el efecto sobre una estructura terrestre de una perturbación de tipo 
terremoto. Definiendo a la estructura en reposo en $t=0$, y sea $X(t)>0, t \geq 0$, el desplazamiento (relativo) horizontal del techo con respecto al suelo. Basándose en un modelo lineal idealizado, el desplazamiento relativo $\mathrm{X}(\mathrm{t})$ se rige por la ecuación diferencial.

$\left\{\begin{array}{l}X^{\prime \prime}(t)+2 \backslash \xi \omega_{0} \dot{X}(t)+\omega_{0}^{2} X(t)=-Y(t), \quad t \geq 0 \\ X(0)=0, \dot{X}(0)=0\end{array}\right.$

Donde $\mathrm{Y}(\mathrm{t})$ es el proceso estocástico de segundo orden dado por (1.1). La solución en media cuadrática tiene la forma:

$X(t)=-\int_{0}^{t} h(t-z) Y(z) d z$

con $\xi<1$, y donde $\mathrm{h}(\mathrm{t})$ es la respuesta impulsiva

$$
\begin{aligned}
& h(t)=\frac{1}{\omega_{0}} e^{-\backslash \xi \omega_{0} t} \sin \left(\widehat{\omega_{0}} t\right), t \geq 0, \widehat{\omega_{0}}=\omega_{0} \sqrt{1-\backslash \xi^{2}} \\
& \Gamma_{Y}(u, v)=E[Y(u) Y(v)]=\frac{1}{2} \sum_{j=1}^{m} u v a_{j}^{2} e^{-\alpha_{j}(u+v)} \cos \left((u-v) \omega_{j}\right), u, v \geq 0
\end{aligned}
$$

Por lo tanto, de las ecuaciones (1.2)-(1.4), se deduce que $\mathrm{E}[\mathrm{X}(\mathrm{t})]=0, \mathrm{t} \geq 0$

$$
\operatorname{Var}[X(t)]=E[X(t) X(t)]=\int_{0}^{t} \int_{0}^{t} h(t-u) h(t-v) \Gamma_{Y}(u, v) d u d v, t \geq 0
$$

Para aplicar el esquema aleatorio de Euler al problema, se convierte la ecuación diferencial de segundo orden en un sistema de ecuaciones diferenciales de primer orden. Dejando $\mathrm{X}^{1}(\mathrm{t})=\mathrm{X}(\mathrm{t}), \mathrm{X}^{2}(\mathrm{t})=\dot{\mathrm{X}}(\mathrm{t})$ y $(\mathrm{X}(\mathrm{t}))^{\mathrm{T}}=\left[\mathrm{X}^{1}(\mathrm{t}), \mathrm{X}^{2}(\mathrm{t})\right]$, la forma de la matriz vectorial de la ecuación (1.1) es

$$
\dot{X}(t)=A(t) X(t)+G(t)
$$

Donde $A(t)$ y $G(t)$ se han obtenido en $(1.15)$ y $(X(0))^{T}=0^{T}=[0,0]$. Como $X_{0}^{T}=[0,0]$, la expresión del método aleatorio de Euler en este caso toma la forma.

$$
X_{n}=(I+h A)^{n} \mathbf{X}_{\mathbf{0}}+h \sum_{i=0}^{n-1}(I+h A)^{n-i-1} \mathbf{G}\left(t_{i}\right)=h \sum_{i=0}^{n-1}(I+h A)^{n-i-1} \mathbf{G}\left(t_{i}\right)
$$

Donde la matriz de identidad de tamaño 2, las hipótesis H1 y H2 se cumplen, luego la convergencia en media cuadrática del método aleatorio de Euler está garantizada. De hecho, H1 se cumple, y se obtiene la condición de Lipschitz.

$$
|| \mathrm{F}(\mathbf{X}, \mathrm{t})-\left.\mathrm{F}(\mathbf{Y}, \mathrm{t})\right|_{r \times s} \leq|| \mathrm{A} \|\left.|\infty||\mathrm{X}-\mathrm{Y}|\right|_{r \times s}=\mathrm{k}|| \mathrm{X}-\mathrm{Y}||_{\mathrm{r} \times \mathrm{s}},
$$


Considerando que $\mathrm{E}\left[\mathrm{Y}\left(\mathrm{t}_{\mathrm{i}}\right)\right]=0$, se obtiene que $\mathrm{E}\left[\mathrm{X}_{\mathrm{n}}\right]=0$ y $\mathrm{E}\left[\mathrm{X}_{\mathrm{n}}\right]\left(\mathrm{E}\left[\mathrm{X}_{\mathrm{n}}\right]\right)^{\mathrm{T}}=0$, luego la matriz de covarianza del vector aleatorio $\mathbf{X}_{\mathbf{n}}$ será:

$$
\begin{aligned}
& \Lambda_{\mathrm{X}_{\mathrm{n}}}=\mathrm{E}\left[\mathrm{X}_{\mathrm{n}}\left(\mathrm{X}_{\mathrm{n}}\right)^{\mathrm{T}}\right] \\
& \left.=\mathrm{h}^{2} \sum_{\mathrm{i}=0}^{\mathrm{n}-1} \sum_{\mathrm{j}=0}^{\mathrm{n}-1}(\mathrm{I}+\mathrm{hA})^{\mathrm{n}-\mathrm{i}-1} \mathrm{E}[\mathrm{G}(\mathrm{ti}) \mathrm{G}(\mathrm{tj}))^{\mathrm{T}}\right]\left((\mathrm{I}+h \mathbf{A})^{n-i-1}\right)^{T}
\end{aligned}
$$

Siendo

$$
\mathrm{E}\left[\mathrm{G}\left(\mathrm{t}_{\mathrm{i}}\right)\left(\mathrm{G}\left(\mathrm{t}_{\mathrm{j}}\right)\right)^{\mathrm{T}}\right]=\left[\begin{array}{cc}
0 & 0 \\
0 & \mathrm{E}\left[\mathrm{Y}\left(t_{i}\right) \mathrm{Y}\left(t_{j}\right)\right.
\end{array}\right]
$$

Donde $\mathrm{E}\left[\mathrm{Y}\left(\mathrm{t}_{\mathrm{i}}\right) \mathrm{Y}\left(\mathrm{t}_{\mathrm{j}}\right)\right]$ está definido por (1.5). Teniendo en cuenta que si $\Lambda_{\mathrm{X}_{\mathrm{n}}}=\left(\mathrm{V}^{\mathrm{ij}}\right)_{2 \times 2}$, denota la matriz cuadrada de tamaño 2 dada por (1.6), entonces $\mathrm{V}^{11}$ es la varianza aproximada del proceso estocástico de segundo orden (1.1), obtenida del método aleatorio escalar de Euler.

\subsection{Problema matricial lineal bilateral completo de Sylverter}

Se considera el sistema diferencial aleatorio matricial no homogéneo dado por

$$
\left\{\begin{array}{l}
\dot{X}(t)=A(t) X(t)+X(t) B(t)+C(t), t \geq 0, \\
X(0)=X_{0},
\end{array}\right.
$$

Donde

$$
\mathrm{A}(\mathrm{t})=\left[\begin{array}{ll}
0 & 1 \\
1 & 0
\end{array}\right], B(t)=\left[\begin{array}{ll}
1 & 1 \\
0 & 0
\end{array}\right], C(t)=\left[\begin{array}{cc}
B(t) & 1 \\
0 & (B(t))^{2}
\end{array}\right], X_{0}=0
$$

Siendo 0 la matriz nula de tamaño $2 \times 2$ y $B(t)$ un proceso de movimiento Browniano. Ya que $\mathrm{E}\left[(\mathrm{B}(\mathrm{t}))^{2}\right]=\mathrm{ty}\left[(\mathrm{B}(\mathrm{t}))^{4}\right]=3 \mathrm{t}^{2}$, entonces para $0 \leq \mathrm{t} \leq \mathrm{t}^{\prime}$ resulta que

$$
\begin{aligned}
|| F(X, t)-F\left(X, t^{\prime}\right) \|\left.\right|_{r \times s}= & \max \left\{\left\|B(t)-B\left(t^{\prime}\right)\right\|,\left\|(B(t))^{2}-\left(B\left(t^{\prime}\right)\right)^{2}\right\|\right\} \\
& \max \left\{\left|t-t^{\prime}\right|^{1 / 2},\left|-t^{2}+3\left(t^{\prime}\right)^{2}-2 t^{\prime}\right|^{1 / 2}\right\},
\end{aligned}
$$

y por el Lema 1.0.1 se obtiene

$$
\begin{aligned}
\|\mathbf{F}(\mathbf{X}, t)-\mathbf{F}(\mathbf{X}, t)\|_{r \times s} & \leq\|\mathbf{A}(\mathbf{X}-\mathbf{Y})\|_{r \times s}+\|(\mathbf{X}-\mathbf{Y}) \mathbf{B}\|_{r \times s} \\
& \leq\left(\|\mathbf{A}\|_{\infty}+\|\mathbf{B}\|_{\infty}\right)\|\mathbf{X}-\mathbf{Y}\|_{r \times s},
\end{aligned}
$$

La ecuación del problema (1.7) satisface las hipótesis H1 y H2, por lo tanto, el método aleatorio de Euler es convergente en media cuadrática

Denotando por $\mathrm{E}[\mathrm{Xn}]=(\mathrm{Eij}) 2 \times 2$ la esperanza de la matriz de tamaño $2 \times 2$ de las aproximaciones de Euler. 


\subsubsection{Problema escalar lineal con termino fuente Browniano}

$\left\{\begin{array}{l}\dot{X}(\mathrm{t})=2 \mathrm{tX}(\mathrm{t})+\exp (-\mathrm{t})+\mathrm{B}(\mathrm{t}), \mathrm{t} \in[0,1] \\ \mathrm{X}\left(\mathrm{t}_{0}\right)=\mathrm{X}_{0}\end{array}\right.$

Donde $\mathrm{B}(\mathrm{t})$ es el proceso movimiento Browniano estándar y $X_{0}$ es la variable aleatoria, $X_{0} \sim N\left(\frac{\frac{1}{2,1}}{12}\right)$ independiente del movimiento Browniano $B(t)$ para cada $t \in[0,1]$.

Figura 1

Valores numéricos de $E^{11}$ de la matriz esperanza del Problema Matricial

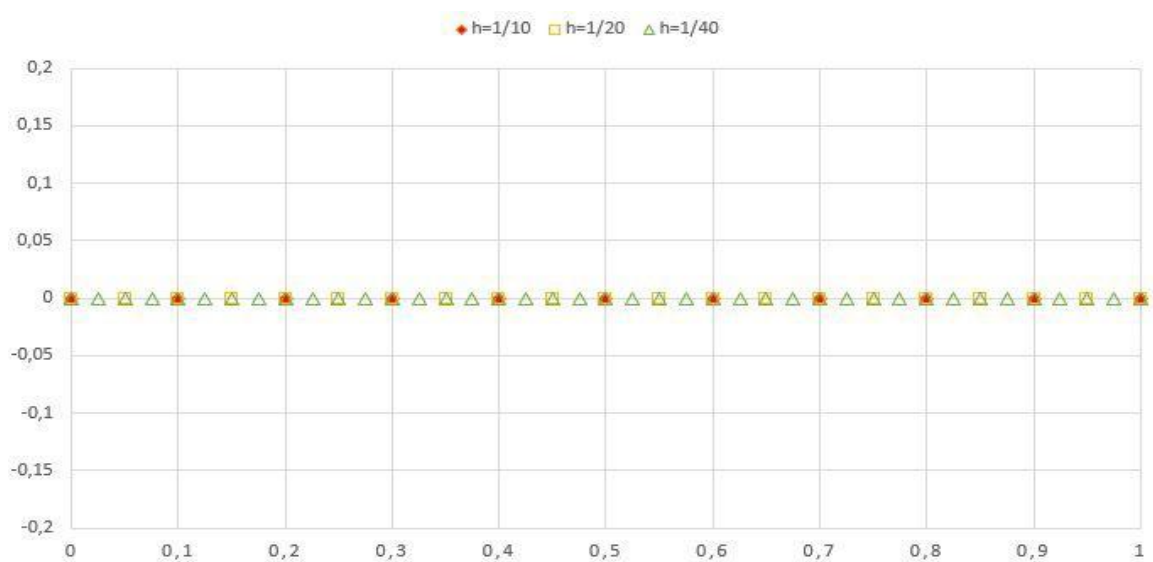

Fuente: El Autor

Figura 2

Valores numéricos de $E^{21}$ de la matriz esperanza del Problema Matricial

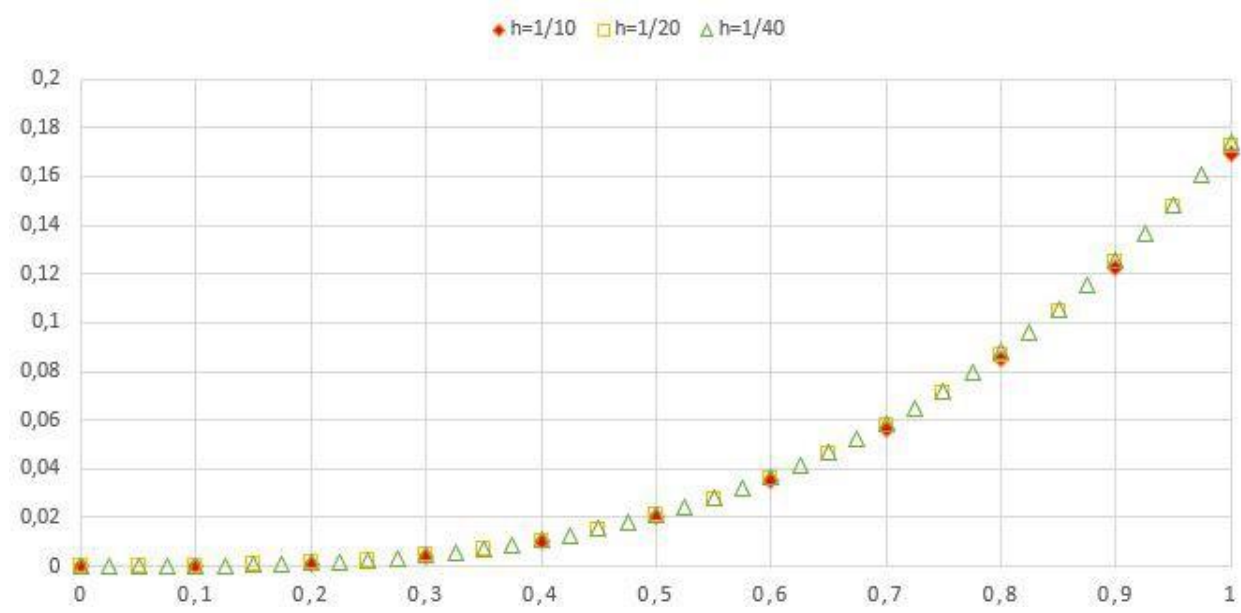

Fuente: El Autor

$$
\mathrm{X}(\mathrm{t})=\exp \left(\mathrm{t}^{2}\right) \backslash\left\{\mathrm{X}_{0}+\int_{0}^{\mathrm{t}} \exp \left(-\mathrm{s}^{2}\right)(\exp (-\mathrm{s})+\mathrm{B}(\mathrm{s})) \mathrm{ds} \backslash\right\}
$$

Se nota que F(X,t) cumple la condición de Lipschitz $\mathbf{H 2}$, ya que 


$$
\begin{aligned}
\left\|F(X, t)-F\left(X^{*}, t\right)\right\| & =\left\|2 t X+\exp (-t)+B(t)-2 t X^{*}-\exp (-t)-B(t)\right\| \\
& =\left\|2 t X-2 t X^{*}\right\|=2 t|| X-X^{*} \|
\end{aligned}
$$

Si $F(X, t)=2 t X+\exp (-t)+B(t)$, entonces

$$
|| F(X, t)-F\left(X, t^{*}\right)|| \leq(2|| X||+1)\left|t-t^{*}\right|+\left|t-t^{*}\right|^{1 / 2}
$$

y $F(X, t)$ esta acotada aleatoriamente, presentándose de manera continua uniforme dentro de cualquier conjunto acotado $\mathrm{S} \subset \mathrm{L}^{2}$. El método aleatorio de cuarto orden de Runge-Kutta es:

$$
X_{n+1}=X_{n}+\frac{1}{6}\left(K_{1}+2 K_{2}+2 K_{3}+K_{4}\right)
$$

Donde

$$
\begin{gathered}
\mathrm{K}_{1}=2 \mathrm{ht}_{\mathrm{n}} \mathrm{X}_{\mathrm{n}}+\mathrm{h}\left(\exp \left(-\mathrm{t}_{-} \mathrm{n}\right)+\mathrm{B}\left(\mathrm{t}_{-} \mathrm{n}\right)\right) \\
\mathrm{K}_{2}=2 \mathrm{~h}\left(\mathrm{t}_{\mathrm{n}}+\frac{\mathrm{h}}{2}\right)\left(1+\mathrm{ht}_{\mathrm{n}}\right) \mathrm{X}_{\mathrm{n}}+\mathrm{h}^{2}\left(\mathrm{t}_{\mathrm{n}}+\frac{h}{2}\right)\left(\exp \left(-\mathrm{t}_{-} \mathrm{n}\right)+\mathrm{B}\left(\mathrm{t}_{\mathrm{n}}\right)\right) \\
+\mathrm{h}\left(\exp \left(-\left(\mathrm{t}_{\mathrm{n}}+\frac{\mathrm{h}}{2}\right)\right)+\mathrm{B}\left(\mathrm{t}_{\mathrm{n}}+\frac{\mathrm{h}}{2}\right)\right), \\
\mathrm{K}_{-} 3=2 \mathrm{~h}\left(\mathrm{t}_{\mathrm{n}}+\frac{\mathrm{h}}{2}\right)\left(1+\mathrm{h}\left(\mathrm{t}_{\mathrm{n}}+\frac{\mathrm{h}}{2}\right)\left(1+\mathrm{ht} \mathrm{n}_{\mathrm{n}}\right)\right) \mathrm{X}_{\mathrm{n}}+\mathrm{h}^{3}\left(\mathrm{t}_{\mathrm{n}}+\frac{\mathrm{h}}{2}\right)^{2}\left(\exp \left(-\mathrm{t}_{\mathrm{n}}\right)+\mathrm{B}\left(\mathrm{t}_{\mathrm{n}}\right)\right) \\
+\mathrm{h}\left(1+\mathrm{h} \backslash \mathrm{bigg}\left(\mathrm{t}_{\mathrm{n}}+\frac{\mathrm{h}}{2}\right)\right)\left(\exp \left(-\left(\mathrm{t}_{\mathrm{n}}+\frac{\mathrm{h}}{2}\right)\right)+\mathrm{B}\left(\mathrm{t}_{\mathrm{n}}+\frac{\mathrm{h}}{2}\right)\right) \\
\mathrm{K}_{4}=2 \mathrm{~h}\left(\mathrm{t}_{\mathrm{n}}+\mathrm{h}\right)\left(1+2 \mathrm{~h}\left(\mathrm{t}_{\mathrm{n}} \frac{h}{2}\right)\left(1+\mathrm{h}\left(\mathrm{t}_{\mathrm{n}}+\frac{\mathrm{h}}{2}\right)\left(1+\mathrm{ht} \mathrm{n}_{\mathrm{n}}\right)\right) \mathrm{X}_{\mathrm{n}}+2 \mathrm{~h}^{4}\left(\mathrm{t}_{\mathrm{n}}+\mathrm{h}\right)\right. \\
\times\left(\mathrm{t}_{\mathrm{n}}+\frac{\mathrm{h}}{2}\right)^{2}\left(\exp \left(-\mathrm{t}_{\mathrm{n}}\right)+\mathrm{B}\left(\mathrm{t}_{\mathrm{n}}\right)\right)+2 \mathrm{~h}^{2}\left(\mathrm{t}_{\mathrm{n}}+\mathrm{h}\right)\left(1+\mathrm{h}\left(\mathrm{t}_{\mathrm{n}}+\frac{\mathrm{h}}{2}\right)\right) \\
\left.\times\left(\exp \left(-\left(t_{n}+\frac{h}{2}\right)\right)\right)+B\left(t_{n}+\frac{h}{2}\right)+h\left(\exp \left(-\left(t_{n}+h\right)\right)\right)+B\left(t_{n}+h\right)\right),
\end{gathered}
$$

\subsection{EI método aleatorio de Euler y su convergencia en media cuadrática}

Consideremos el el problema bajo las siguientes hipótesis $F: S \times T-\rightarrow L_{2}$, con $S \subset L_{2}$ :

1. $\quad \mathrm{H} 1: F(X, t)$ es uniformemente continua sobre ventanas temporales

2. $\quad \mathrm{H} 2: F(X, t)$ satisface la condición de Lipschitz en media cuadrática

$$
|| F(X, t)-F(Y, t) \| \leq k(t)|| X-Y||, \backslash h s p a c e 3 m m \int_{t_{0}}^{t_{e}} k(t) d t<+\infty .
$$

La condición $\mathrm{H} 2$ garantiza la continuidad en media cuadrática de $F(X, t)$ con respecto a la primera variable, mientras que $\mathrm{H} 1$ garantiza la continuidad de $\mathrm{F}(\mathrm{X}, \mathrm{t})$ con respecto a la segunda variable, de este análisis proviene la desigualdad 


$$
|| F(X, t)-F\left(Y, t^{\prime}\right)\|\leq|| F(X, t)-F(Y, t)\|+|| F(Y, t)-F\left(Y, t^{\prime}\right) \|
$$

Se obtiene la continuidad en media cuadrática de $\mathrm{F}(\mathrm{X}, \mathrm{t})$ con respecto a las dos variables. Consideremos el método aleatorio de Euler para el problema de valor inicial

$$
\left\{\begin{array}{l}
X_{n+1}=X_{n}+h F\left(X_{n}, t_{n}\right), \quad n \geq 0 \\
X_{0}=X\left(t_{0}\right)
\end{array}\right.
$$

Donde $\mathrm{h}=\mathrm{t}_{\mathrm{n}}-\mathrm{t}_{\mathrm{n}-1}$, cont $_{\mathrm{n}}=\mathrm{t}_{0}+$, paran $=1,2,3$. Demostraremos que bajo las hipótesis H1 y H2, el método de Euler (2.2) es convergente en media cuadrática en el sentido de estación fija, i.e. el error de la media cuadrática.

$e_{n}=X_{n}-X(t)=X_{n}-X\left(t_{n}\right)$

Tiende a cero en L2, cuando $h \rightarrow 0, n \rightarrow$ cont $-t_{0}=n h$.

Bajo las hipótesis H1 y H2, el Teorema 5.1.2 garantiza la existencia y unicidad de la solución media cuadrática $X(t) e n\left[t_{n}, t_{n+1}\right] \subset\left[t_{0}, t_{e}\right]$, y por el Teorema 1.0.2, deducimos.

$X\left(t_{n+1}\right)=X\left(t_{n}\right)+\int_{t_{n}}^{t_{n+1}} \dot{X}(u) d u, n \geq 0$

SX es un conjunto acotado en L2 definido por la solución teórica exacta del problema de valor inicial, $\quad \mathrm{S}_{\mathrm{X}}=\left\{\mathrm{X}(\mathrm{t}), \mathrm{t}_{0} \leq \mathrm{t} \leq \mathrm{t}_{\mathrm{e}}\right\}$

Luego, por la hipótesis H1 y la Definición 1.0.1, obtenemos ||$F\left(X(u), t_{n}\right)-F(X(u), u) \| \leq \omega\left(S_{X}, h\right)$

y por lo tanto, toma la forma

||$e_{n+1}\left\|\leq|| e_{n}\right\|\left[1+h k\left(t_{n}\right)\right]+h\left[\omega\left(S_{X}, h\right)+h M_{\dot{X}} k\left(t_{n}\right)\right]$

Obtenemos:

$$
|| e_{n}\left\|\leq e^{n h k\left(t_{n}\right)}|| e_{0}\right\|+\frac{e^{n h k\left(t_{n}\right)}-1}{k\left(t_{n}\right)}\left[\omega\left(S_{X}, h\right)+h M_{\dot{X}} k(t)\right],
$$

y como $\mathrm{nh}=\mathrm{t}-\mathrm{t},|| \mathrm{e}_{0}||=0$, la anterior inecuación se escribe como

$\left\|\mathrm{e}_{\mathrm{n}}\right\| \leq \frac{\mathrm{e}^{\left(\mathrm{t}-\mathrm{t}_{\mathrm{o}}\right) \mathrm{k}(\mathrm{t})}-1}{\mathrm{k}(\mathrm{t})}\left[\omega\left(\mathrm{S}_{\mathrm{X}}, \mathrm{h}\right)+\mathrm{hM}_{\dot{\mathrm{X}}} \mathrm{k}(\mathrm{t})\right]$

De (2.8), deducimos que $\left\{\mathrm{e}_{\mathrm{n}}\right\}$ es convergente a cero en media cuadrática.

Teorema 2.1.1. Bajo las hipótesis $\mathrm{H} 1$ y H2, el método aleatorio de Euler (2.2) es convergente en media cuadrática y el error de discretización en, definido por (2.3), satisface la desigualdad (2.8) para $\mathrm{t}=\mathrm{t}_{0}+\mathrm{nh}, \mathrm{h}>0, \mathrm{t}_{0} \leq \mathrm{t} \leq \mathrm{t}_{\mathrm{e}}$

\subsection{Estudio del esquema de Runge-Kutta para ecuaciones diferenciales aleatorias}

Los métodos numéricos son metodologías que utilizan técnicas meramente algebraicas y 
aritméticas para resolver de forma aproximada ecuaciones o sistemas de ecuaciones complejos, que analíticamente resultan muy difíciles e incluso imposible de resolver. Los métodos Runge-Kutta, extienden esta idea geométrica utilizando varias derivadas o tangentes intermedias en lugar de solo una, para aproximar la función desconocida. En concreto, un método Runge-Kutta explícito de s etapas se puede escribir siguiendo la siguiente estructura:

$$
\begin{gathered}
\mathrm{K}_{1}=h \mathrm{~F}\left(\mathrm{t}_{\mathrm{n}}, \mathrm{X}_{\mathrm{n}}\right), \\
\mathrm{K}_{2}=h \mathrm{~F}\left(\mathrm{t}_{\mathrm{n}}+\mathrm{c}_{2} \mathrm{~h}, \mathrm{X}_{\mathrm{n}}+\mathrm{a}_{2,1} \mathrm{~K}_{1}\right), \\
\mathrm{K}_{3}=\mathrm{hF}\left(\mathrm{t}_{\mathrm{n}}+\mathrm{c}_{3} \mathrm{~h}, \mathrm{X}_{\mathrm{n}}+\left(\mathrm{a}_{3,1} \mathrm{~K}_{1}+\mathrm{a}_{3,2} \mathrm{~K}_{2}\right)\right) \\
\mathrm{K}_{4}=\mathrm{hF}\left(\mathrm{t}_{\mathrm{n}}+\mathrm{c}_{4} \mathrm{~h}, \mathrm{X}_{\mathrm{n}}+\left(\mathrm{a}_{4,1} \mathrm{~K}_{1}+\mathrm{a}_{4,3} \mathrm{~K}_{3}+\mathrm{a}_{4,2} \mathrm{~K}_{2}\right)\right) \\
\mathrm{K}_{\mathrm{s}}=\mathrm{hF}\left(\mathrm{t}_{\mathrm{n}}+\mathrm{c}_{\mathrm{s}} \mathrm{h}, \mathrm{X}_{\mathrm{n}}+\sum_{\mathrm{j}=1}^{\mathrm{s}-1} \mathrm{a}_{\mathrm{s}, \mathrm{j}} \mathrm{K}_{\mathrm{j}}\right)
\end{gathered}
$$

Donde los cálculos se van realizando de forma recursiva. De

$\mathrm{X}_{\mathrm{n}+1}=\mathrm{X}_{\mathrm{n}}+\frac{1}{6}\left(\mathrm{~K}_{1}+2 \mathrm{~K}_{2}+2 \mathrm{~K}_{3}+\mathrm{K}_{4}\right), \mathrm{n}=0,1,2, \ldots$

En particular, el método de Euler dado en (2.2) se puede escribir como un método de Runge- Kutta de 1 etapa y por tanto requerirá en cada paso de una evaluación de la función F. En particular, en este capítulo vamos a centrarnos en el método estándard Runge-Kutta de orden 4, que posiblemente se trata del método Runge-Kutta más conocido y utilizado al mantener un buen equilibrio entre coste computacional y precisión. Se trata de un método cuyo orden de convergencia global es 4.

Teorema 3.0.1. Consideremos $\mathrm{F}(\mathrm{X}(\mathrm{t}), \mathrm{t})$, definida de $\mathrm{S} \times \mathrm{I}$ en $\mathrm{L}_{2}$, donde $S$ es un conjunto acotado en $\mathrm{L}_{2}$. Si $\mathrm{F}(\mathrm{X}(\mathrm{t}), \mathrm{t})$ satisface las siguientes condiciones

$\left(\mathrm{C}_{1}\right) \mathrm{F}(\mathrm{X}, \mathrm{t})$ es aleatoria uniformemente continua y acotada.

$\left(C_{2}\right) F(X, t)$ satisface la condición de Lipschitz en media cuadrática, es decir, $\|\mathrm{F}(\mathrm{X}, \mathrm{t})-\mathrm{F}(\mathrm{Y}, \mathrm{t})\| \leq \mathrm{K}(\mathrm{t})\|\mathrm{X}-\mathrm{Y}\|$

Donde

$$
\int_{T_{0}}^{T} K(t) d t<\infty
$$

entonces, el esquema aleatorio de Runge-Kutta de cuarto orden (3.2) es convergente en media cuadrática.

Aplicando la regla de L'Hôpital se obtiene

$$
\lim _{h \rightarrow 0} \frac{\mathrm{h}\left(1-\mathrm{e}^{-\mathrm{KT}} \mathrm{e}^{-\mathrm{Kh}}\right)}{1-\mathrm{e}^{-\mathrm{Kh}}}=\left\{\frac{0}{0}\right\}=\lim _{\mathrm{h} \rightarrow 0} \frac{\left(1-\mathrm{e}^{-\mathrm{KT}} \mathrm{e}^{-\mathrm{Kh}}+\mathrm{hK} \mathrm{e}^{-\mathrm{KT}} \mathrm{e}^{-\mathrm{Kh}}\right\}}{\mathrm{Ke}^{-\mathrm{Kh}}}=\frac{1-\mathrm{e}^{-\mathrm{KT}}}{\mathrm{K}}
$$

Por otra parte,

$\lim _{h \rightarrow 0}\left(\frac{3}{2} M h K+\omega(S, h)\right)=0$

ya que $\omega(S, h) \rightarrow 0$ cuando $h \rightarrow 0$. 
Por tanto, tomando límites cuando $\mathrm{h} \rightarrow 0, \mathrm{n} \rightarrow \infty$ con $\mathrm{nh}=\mathrm{T}$, se deduce que en || $\mathrm{e}_{\mathrm{n}+1} \| \rightarrow$ 0 cuando $\mathrm{n} \rightarrow 0$ lo que prueba la convergencia del método de Runge-Kutta.

\subsection{Determinación del desplazamiento por un movimiento terrestre}

Dentro de la determinación del efecto sobre una estructura terrestre de una perturbación de tipo terremoto. Supongamos que la estructura está en reposo en $t=0$, y sea $X(t)>$ $0, t \geq 0$, el desplazamiento (relativo) horizontal del techo con respecto al suelo. Basándose en un modelo lineal idealizado, el desplazamiento relativo $\mathrm{X}(\mathrm{t})$ se rige por la ecuación diferencial

$$
\left\{\begin{array}{l}
X(t)+2 \backslash \xi \omega_{0} \dot{X}(t)+\omega_{0}^{2} X(t)=-Y(t), \quad t \geq 0 \\
X(0)=0, \dot{X}(0)=0
\end{array}\right.
$$

donde $\mathrm{Y}(\mathrm{t})$ es el proceso estocástico de segundo orden dado por (1.16). La solución en media cuadrática del problema (4.1) tiene la forma:

$\mathrm{X}(\mathrm{t})=-\int_{0}^{\mathrm{t}} \mathrm{h}(\mathrm{t}-\mathrm{z}) \mathrm{Y}(\mathrm{z}) \mathrm{dz}$

Con $\xi<1$, y donde $h(t)$ es la respuesta impulsiva

$h(t)=\frac{1}{\widehat{\omega_{0}}} e^{-\backslash \xi \omega_{0} t} \sin \left(\widehat{\omega_{0}} t\right), t \geq 0, \widehat{\omega_{0}}=\omega_{0} \sqrt{1-\backslash \xi^{2}}$

La esperanza y la función de autocorrelación del proceso estocástico de segundo orden $\mathrm{Y}(\mathrm{t})$ están dadas por

$\mathrm{E}[\mathrm{Y}(\mathrm{t})]=0, \mathrm{t} \geq 0$

$\mathrm{Y}$

$\Gamma_{Y}(u, v)=E[Y(u) Y(v)]=\frac{1}{2} \sum_{j=1}^{m} u v a_{j}^{2} e^{-\alpha_{j}(u+v)} \cos \left((u-v) \omega_{j}\right), u, v \geq 0$

Por lo tanto, de las ecuaciones (4.2)-(4.5), se deduce que

$$
\begin{gathered}
\mathrm{E}[\mathrm{X}(\mathrm{t})]=0, \quad \mathrm{t} \geq 0 \\
\mathrm{Y} \quad \operatorname{Var}[\mathrm{X}(\mathrm{t})]=\mathrm{E}[\mathrm{X}(\mathrm{t}) \mathrm{X}(\mathrm{t})]=\int_{0}^{\mathrm{t}} \int_{0}^{\mathrm{t}} \mathrm{h}(\mathrm{t}-\mathrm{u}) \mathrm{h}(\mathrm{t}-\mathrm{v}) \Gamma_{\mathrm{Y}}(\mathrm{u}, \mathrm{v}) \mathrm{dudv}, \mathrm{t} \geq 0
\end{gathered}
$$

Dejando $X^{1}(t)=X(t), X^{2}(t)=\dot{X}(t) \quad y(X(t))^{T}=\left[X^{1}(t), X^{2}(t)\right]$, la forma de la matriz vectorial de la ecuación (4.1) es

$\dot{X}(t)=A(t) X(t)+G(t)$,

donde $\mathrm{A}(\mathrm{t})$ y $\mathrm{G}(\mathrm{t})$, se han obtenido en $(1.15)$ y $(\mathrm{X}(0))^{\mathrm{T}}=0^{\mathrm{T}}=[0,0]$. Como $\mathrm{X}_{0}^{\mathrm{T}}=[0,0]$, la expresión del método aleatorio de Euler en este caso toma la forma 


$$
X_{n}=(I+h A)^{n} X_{0}+h \sum_{i=0}^{n-1}(I+h A)^{n-i-1} \mathbf{G}\left(t_{i}\right)=h \sum_{i=0}^{n-1}(I+h A)^{n-i-1} \mathbf{G}\left(t_{i}\right)
$$

Donde se puede denotar la matriz de identidad de tamaño 2. Señalando que las hipótesis H1 y H2 se cumplen, luego la convergencia en media cuadrática del método aleatorio de Euler está garantizada. De hecho, H1 se cumple con el Ejemplo 1.0.2 y, según el Lema 1.0.1, se obtiene la condición de Lipschitz.

$$
|| F(X, t)-\left.F(Y, t)\right|_{r \times s} \leq|| A||_{\infty}|| X-Y||_{r \times s}=\mathrm{k}|| X-Y||_{r \times s}
$$

Donde

$$
\mathrm{k}=\max \left\{1, \omega_{0}^{2}+2 \omega_{0} \xi\right\}, \int_{\mathrm{t}_{0}}^{\mathrm{t}_{\mathrm{e}}} \mathrm{kdt}<+\infty
$$

Teniendo en cuenta que como $\mathrm{E}\left[\mathrm{Y}\left(\mathrm{t}_{\mathrm{i}}\right)\right]=0$, obtenemos que $\mathrm{E}\left[\mathrm{X}_{\mathrm{n}}\right]=0$ y $\mathrm{E}\left[\mathrm{X}_{\mathrm{n}}\right]\left(\mathrm{E}\left[\mathrm{X}_{\mathrm{n}}\right]\right)^{\mathrm{T}}=$ 0 , luego la matriz de covarianza del vector aleatorio $\mathbf{X}_{\mathrm{n}}$ sería

$$
\begin{aligned}
& \Lambda_{\mathrm{X}_{\mathrm{n}}}=\mathrm{E}\left[\mathrm{X}_{\mathrm{n}}\left(\mathrm{X}_{\mathrm{n}}\right)^{\mathrm{T}}\right] \\
& =h^{2} \sum_{i=0}^{n-1} \sum_{j=0}^{n-1}(I+h A)^{n-i-1} E\left[\left(G\left(t_{i}\right) G\left(t_{j}\right)\right)^{T}\right]\left((I+h A)^{n-j-1}\right)^{T} \\
& E\left[G\left(t_{i}\right)\left(G\left(t_{j}\right)\right)^{T}\right]= \\
& E\left[G\left(t_{i}\right)\left(G\left(t_{j}\right)\right)^{T}\right]=\left[\begin{array}{cc}
0 & 0 \\
0 & \mathrm{E}\left[\mathrm{Y}\left(t_{i}\right) \mathrm{Y}\left(t_{j}\right)\right.
\end{array}\right]
\end{aligned}
$$

Donde $\mathrm{E}\left[\mathrm{Y}\left(\mathrm{t}_{\mathrm{i}}\right) \mathrm{Y}\left(\mathrm{t}_{\mathrm{j}}\right)\right]$ está definido por (4.5). Teniendo en cuenta que si $\Lambda_{\mathrm{X}_{\mathrm{n}}}=\left(\mathrm{V}^{\mathrm{ij}}\right)_{2 \times 2}$, denota la matriz cuadrada de tamaño 2 dada por (4.7), entonces $\mathrm{V}^{11}$ es la varianza aproximada del proceso estocástico de segundo orden (4.1), obtenida del método aleatorio escalar de Euler. La Figura 4.1 muestra $\mathrm{V}^{11}=\operatorname{Var}\left[\mathrm{X}_{\mathrm{n}}\right]$

Para $\mathrm{h}=1 / 20, \mathrm{~h}=1 / 40, \mathrm{~h}=1 / 80$ y la varianza teórica $\operatorname{Var}\left[\mathrm{X}\left(\mathrm{t}_{\mathrm{n}}\right)\right]$ dado por (4.6), tomando en (4.1) $\xi=0,05 \mathrm{y} \omega_{\mathrm{j}}=1, \mathrm{a}_{\mathrm{j}}=(1 / 2)^{\mathrm{j}}, \$ 0 \backslash$ leq $\mathrm{j} \backslash$ leq 20 para $\mathrm{Y}(\mathrm{t})$ dado por (1.8). Dicha gráfica ilustra, que los valores numéricos y exactos están más cerca cuando $\mathrm{h}$ disminuye. Este comportamiento está de acuerdo con la propiedad (1.9).

\subsection{Problema de ruido blanco Gaussiano}

Consideremos el problema

$$
\left[\begin{array}{l}
X(t)=t^{2} X(t)+W(t), t \in[0,1] \\
X(0)=X_{-} 0
\end{array}\right.
$$

Donde $W(t)$ es un proceso de ruido blanco Gaussiano con media cero y $X_{0}$ es una variable exponencial aleatoria con el parámetro $\lambda=\frac{1}{2}$, independiente de $W(t)$ para cada $t \in[0,1]$. En este ejemplo tenemos $F(X, t)=t^{2} X+W(t)$. 
La covarianza de $W(t)$ es

$\operatorname{Cov}[\mathrm{W}(\mathrm{t}), \mathrm{W}(\mathrm{s})]=\delta(\mathrm{t}-\mathrm{s})$

Donde $\delta(t)$ es la función delta generalizada. Siempre existe una convolución con la función delta y juega el mismo papel para la convolución que la unidad para la multiplicación, esto es,

$$
\delta * \mathrm{~g}=\mathrm{g}
$$

Tomando $\mathrm{g}(\mathrm{s})=\mathrm{h}(\mathrm{s}) \mathcal{X}[0, \mathrm{t}](\mathrm{s})$, donde $\mathrm{h}(\mathrm{s})$ es una función $\mathrm{C}^{\infty} \mathrm{yX}[0, \mathrm{t}]$ (s) denota la función característica de la función en el intervalo $[0, t]$, de (4.9) se tiene que

$$
\int_{-\infty}^{\infty} \mathrm{g}(\mathrm{s}) \delta(\mathrm{s}-\mathrm{r}) \mathrm{ds}=\int_{-\infty}^{\infty} \mathrm{h}(\mathrm{s}) \mathcal{X}[0, \mathrm{t}](\mathrm{s}) \delta(\mathrm{s}-\mathrm{r}) \mathrm{ds}=\int_{0}^{\mathrm{t}} \mathrm{h}(\mathrm{s}) \delta(\mathrm{s}-\mathrm{r}) \mathrm{ds}=\mathrm{h}(\mathrm{r})
$$

Para calcular la solución exacta del problema, multiplicaremos ambos lados del resultante de la expresión (4.9) por exp $\left(\frac{-t^{3}}{3}\right)$ y usando $W(t)=\frac{d B(t)}{d t}$ tenemos

$$
-t^{2} \exp \left(\frac{-t^{3}}{3}\right) X(t) d t+\exp \left(\frac{-t^{3}}{3}\right) d X(t)=\exp \left(\frac{-t^{3}}{3}\right) d B(t)
$$

y entonces

$X(t)=\exp \left(\frac{-t^{3}}{3}\right)\left[X_{0}+\int_{0}^{t} \exp \left(\frac{-s^{3}}{3}\right) d B(s)\right]$

Ahora, calculamos las aproximaciones $\mathrm{X}_{\mathrm{n}}$ usando el método aleatorio de Runge-Kutta de cuarto orden,

$\mathrm{X}_{\mathrm{n}+1}=\mathrm{X}_{\mathrm{n}}+\frac{1}{6}\left(\mathrm{k}_{1}+2 \mathrm{k}_{2}+2 \mathrm{k}_{3}+\mathrm{k}_{4}\right)$

Donde $\quad \mathrm{k}_{1}=\mathrm{ht}_{\mathrm{n}}^{2} \mathrm{X}_{\mathrm{n}}+\mathrm{hW}\left(\mathrm{t}_{\mathrm{n}}\right)$,

$\mathrm{k}_{2}=\mathrm{h}\left(\mathrm{t}_{\mathrm{n}}+\frac{\mathrm{h}}{2}\right)^{2}\left(1+\frac{\mathrm{h}}{2} \mathrm{t}_{\mathrm{n}}^{2}\right) \mathrm{X}_{\mathrm{n}}+\frac{\mathrm{h}^{2}}{2}\left(\mathrm{t}_{\mathrm{n}}+\frac{\mathrm{h}}{2}\right)^{2} \mathrm{~W}\left(\mathrm{t}_{\mathrm{n}}\right)+\mathrm{hW}\left(\mathrm{t}_{\mathrm{n}}+\frac{\mathrm{h}}{2}\right)$,

$\mathrm{k}_{3}=\mathrm{h}\left(\mathrm{t}_{\mathrm{n}}+\frac{\mathrm{h}}{2}\right)^{2}\left(1+\frac{\mathrm{h}}{2}\left(\mathrm{t}_{\mathrm{n}}+\frac{\mathrm{h}}{2}\right)^{2}\left(1+\frac{\mathrm{h}}{2} \mathrm{t}_{\mathrm{n}}^{2}\right)\right) \mathrm{X}_{\mathrm{n}}+\frac{\mathrm{h}^{3}}{4}\left(\mathrm{t}_{\mathrm{n}}+\frac{\mathrm{h}}{2}\right)^{4} \mathrm{~W}\left(\mathrm{t}_{\mathrm{n}}\right)$,

$\mathrm{k}_{4}=\mathrm{h}\left(\mathrm{t}_{\mathrm{n}}+\mathrm{h}\right)^{2}\left(1+\mathrm{h}\left(\mathrm{t}_{\mathrm{n}}+\frac{\mathrm{h}}{2}\right)^{2}\left(1+\frac{\mathrm{h}}{2}\left(\mathrm{t}_{\mathrm{n}}+\frac{\mathrm{h}}{2}\right)^{2}\left(1+\frac{\mathrm{h}}{2} \mathrm{t}_{\mathrm{n}}^{2}\right)\right)\right) \mathrm{X}_{\mathrm{n}}+\frac{\mathrm{h}^{4}}{4}\left(\mathrm{t}_{\mathrm{n}}+\frac{\mathrm{h}}{2}\right)^{4}$,

$\times\left(t_{n}+h\right)^{2} W\left(t_{n}\right)+h^{2}\left(t_{n}+h\right)^{2}\left(1+\frac{h}{2}\left(t_{n}+\frac{h}{2}\right)^{2}\right) W\left(t_{n}+\frac{h}{2}\right)+h W\left(t_{n}+h\right)$,

Tenemos

$$
X_{n+1}=a_{n} X_{n}+b_{n}, n=0,1,2, \ldots,
$$

$\mathrm{y}$ procediendo de forma recurrente obtenemos

$X_{n}=\left(\prod_{i=0}^{n-1} a_{i}\right) X_{0}+\sum_{i=0}^{n-1}\left(\prod_{j=i+1}^{n-1} a_{j}\right) b_{i}, n=1,2,3, \ldots$

Donde

$\mathrm{E}\left[\mathrm{b}_{\mathrm{i}} \mathrm{b}_{\mathrm{k}}\right]=\mathrm{A}_{\mathrm{i}, \mathrm{k}} \delta\left(\mathrm{t}_{\mathrm{i}}-\mathrm{t}_{\mathrm{k}}\right)+\mathrm{B}_{\mathrm{i}, \mathrm{k}} \delta\left(\mathrm{t}_{\mathrm{i}}-\mathrm{t}_{\mathrm{k}}-\frac{\mathrm{h}}{2}\right)+\mathrm{B}_{\mathrm{i}, \mathrm{k}} \delta\left(\mathrm{t}_{\mathrm{i}}-\mathrm{t}_{\mathrm{k}}+\frac{\mathrm{h}}{2}\right)+\mathrm{C}_{\mathrm{i}} \delta\left(\mathrm{t}_{\mathrm{i}}-\mathrm{t}_{\mathrm{k}}-\mathrm{h}\right)+$ $\mathrm{C}_{\mathrm{k}} \delta\left(\mathrm{t}_{\mathrm{i}}-\mathrm{t}_{\mathrm{k}}+\mathrm{h}\right), \quad$ con 


\section{Cuadro 1}

Error absoluto de la esperanza de X(t) con Euler y Runge-Kutta de orden 4 del Problema de ruido blanco Gaussiano

\begin{tabular}{ccccc}
\hline t & \multicolumn{2}{c}{ Euler } & \multicolumn{2}{c}{ Runge-Kutta de orden 4 } \\
\cline { 2 - 5 } & $h=\underline{1}$ & $h=\frac{1}{50}$ & $h=\frac{1}{20}$ & $h=\frac{1}{50}$ \\
\hline 0,1 & $8,3335 \times 10^{-5}$ & $2,1868 \times 10^{-4}$ & $2,1709 \times 10^{-10}$ & $2,2227 \times 10^{-12}$ \\
0,2 & $1,8389 \times 10^{-3}$ & $4,2813 \times 10^{-3}$ & $4,3522 \times 10^{-10}$ & $4,4555 \times 10^{-12}$ \\
0,3 & $2,9353 \times 10^{-3}$ & $1,8305 \times 10^{-2}$ & $6,5759 \times 10^{-10}$ & $6,7342 \times 10^{-12}$ \\
0,4 & $5,9445 \times 10^{-3}$ & $4,9066 \times 10^{-2}$ & $8,9216 \times 10^{-10}$ & $9,1442 \times 10^{-12}$ \\
0,5 & $1,0166 \times 10^{-2}$ & $1,0493 \times 10^{-1}$ & $1,1604 \times 10^{-9}$ & $1,1963 \times 10^{-11}$ \\
0,6 & $1,5858 \times 10^{-2}$ & $1,9749 \times 10^{-1}$ & $1,5255 \times 10^{-9}$ & $1,6213 \times 10^{-11}$ \\
0,7 & $2,3460 \times 10^{-2}$ & $3,4425 \times 10^{-1}$ & $2,1834 \times 10^{-9}$ & $2,5666 \times 10^{-11}$ \\
0,8 & $3,3671 \times 10^{-2}$ & $5,7317 \times 10^{-1}$ & $3,7346 \times 10^{-9}$ & $5,3311 \times 10^{-11}$ \\
0,9 & $4,7596 \times 10^{-2}$ & $9,3076 \times 10^{-1}$ & $7,9493 \times 10^{-9}$ & $1,4031 \times 10^{-10}$ \\
1,0 & $6,6954 \times 10^{-2}$ & 1,4968 & $1,9803 \times 10^{-8}$ & $4,0766 \times 10^{-10}$ \\
\hline
\end{tabular}

Fuente: El Autor

\section{Cuadro 2}

Error absoluto de la varianza de X $(t)$ con Euler y Runge-Kutta de orden 4 del Problema de ruido blanco Gaussiano

\begin{tabular}{lllll}
\hline t & \multicolumn{2}{c}{ Euler } & \multicolumn{2}{c}{ Runge-Kutta de orden 4 } \\
\cline { 2 - 5 } & $h=\frac{1}{20}$ & $h=\frac{1}{50}$ & $h=\frac{1}{20}$ & $h=\frac{1}{50}$ \\
\hline 0,1 & $6,0089 \times 10^{-2}$ & $5,9641 \times 10^{-2}$ & $1,0005 \times 10^{-1}$ & $1,0005 \times 10^{-1}$ \\
0,2 & $1,7228 \times 10^{-1}$ & $1,6825 \times 10^{-1}$ & $2,0080 \times 10^{-1}$ & $2,0080 \times 10^{-1}$ \\
0,3 & $3,3247 \times 10^{-1}$ & $3,2120 \times 10^{-1}$ & $3,0408 \times 10^{-1}$ & $3,0408 \times 10^{-1}$ \\
0,4 & $5,3849 \times 10^{-1}$ & $5,1617 \times 10^{-1}$ & $4,1304 \times 10^{-1}$ & $4,1304 \times 10^{-1}$ \\
0,5 & $7,9087 \times 10^{-1}$ & $7,5330 \times 10^{-1}$ & $5,3239 \times 10^{-1}$ & $5,3239 \times 10^{-1}$ \\
0,6 & 1,0930 & 1,0353 & $6,6898 \times 10^{-1}$ & $6,6898 \times 10^{-1}$ \\
0,7 & 1,4516 & 1,3676 & $8,3267 \times 10^{-1}$ & $8,3267 \times 10^{-1}$ \\
0,8 & 1,8769 & 1,7586 & 1,0380 & 1,0380 \\
0,9 & 2,3832 & 2,2199 & 1,3075 & 1,3075 \\
1,0 & 2,9899 & 2,7663 & 1,6764 & 1,6764 \\
\hline
\end{tabular}

Fuente: El Autor

$$
\begin{aligned}
& A_{i, k}=\frac{h^{2}}{36}(1+ {\left[1+h\left(t_{i}+\frac{h}{2}\right)^{2}+\frac{h^{2}}{2}\left(t_{i}+\frac{h}{2}\right)^{4}\left(1+\frac{h}{2}\left(t_{i}+h\right)^{2}\right)\right] } \\
&\left.\times\left[1+h\left(t_{k}+\frac{h}{2}\right)^{2}+\frac{h^{2}}{2}\left(t_{k}+\frac{h}{2}\right)^{4}\left(1+\frac{h}{2}\left(t_{k}+h\right)^{2}\right)\right]\right) \\
&+ \frac{h^{2}}{9}\left[1+\left(1+\frac{h}{2}\left(t_{i}+\frac{h}{2}\right)^{2}\right)\left(1+\frac{h}{2}\left(t_{i}+h\right)^{2}\right)\right] \\
& \times {\left[1+\left(1+\frac{h}{2}\left(t_{k}+\frac{h}{2}\right)^{2}\right)\left(1+\frac{h}{2}\left(t_{k}+h\right)^{2}\right)\right] } \\
& B_{i, k}=\frac{h^{2}}{18}\left(1+\left(\left(1+\left(1+\frac{h}{2}\left(t_{i}+\frac{h}{2}\right)^{2}\right)\right)\left(1+\frac{h}{2}\left(t_{i}+h\right)^{2}\right)+\left[1+h\left(t_{i}+\frac{h}{2}\right)^{2}+\frac{h^{2}}{2}\left(t_{i}+\frac{h}{2}\right)^{4} \times\right.\right.\right.
\end{aligned}
$$




$$
\begin{aligned}
& \left.\left.\left(1+\frac{\mathrm{h}}{2}\left(\mathrm{t}_{\mathrm{i}}+\mathrm{h}\right)^{2}\right)\right]\left[1+\left(1+\frac{\mathrm{h}}{2}\left(\mathrm{t}_{\mathrm{k}}+\frac{\mathrm{h}}{2}\right)^{2}\right)\left(1+\frac{\mathrm{h}}{2}\left(\mathrm{t}_{\mathrm{k}}+\mathrm{h}\right)^{2}\right)\right]\right) \\
& \mathrm{C}_{\mathrm{i}}=\frac{\mathrm{h}^{2}}{36}\left(1+\mathrm{h}\left(\mathrm{t}_{\mathrm{i}}+\frac{\mathrm{h}}{2}\right)^{2}+\frac{\mathrm{h}^{2}}{2}\left(\mathrm{t}_{\mathrm{i}}+\frac{\mathrm{h}}{2}\right)^{4}\left(1+\frac{\mathrm{h}}{2}\left(\mathrm{t}_{\mathrm{i}}+\mathrm{h}\right)^{2}\right)\right), \mathrm{i}, \mathrm{k}=0,1,2, \ldots, \mathrm{n}-1
\end{aligned}
$$

\section{Figura 3}

Esperanza $X(t)$ mediante el método Runge-Kutta de orden 4 y $h=\frac{1}{20}$ del Problema de ruido blanco Gaussiano

Fuente: El Autor

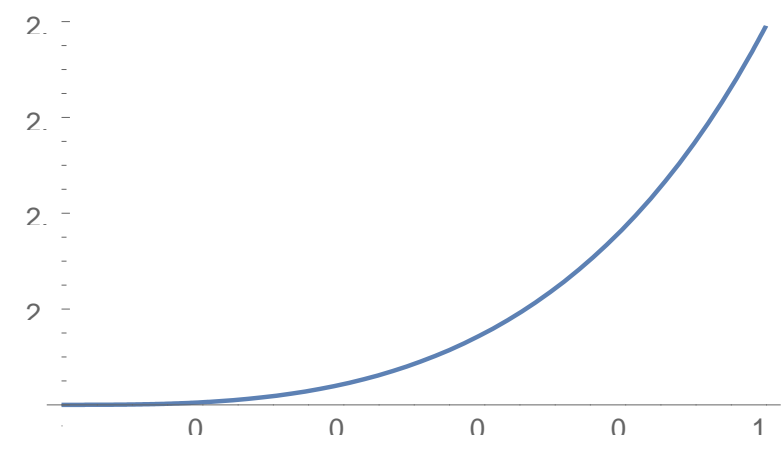

Figura 4

Varianza $X(t)$ y $X_{n}$ mediante el método Runge-Kutta de orden 4 y $h=\frac{1}{20}$ del Problema de ruido blanco Gaussiano

Fuente: El Autor

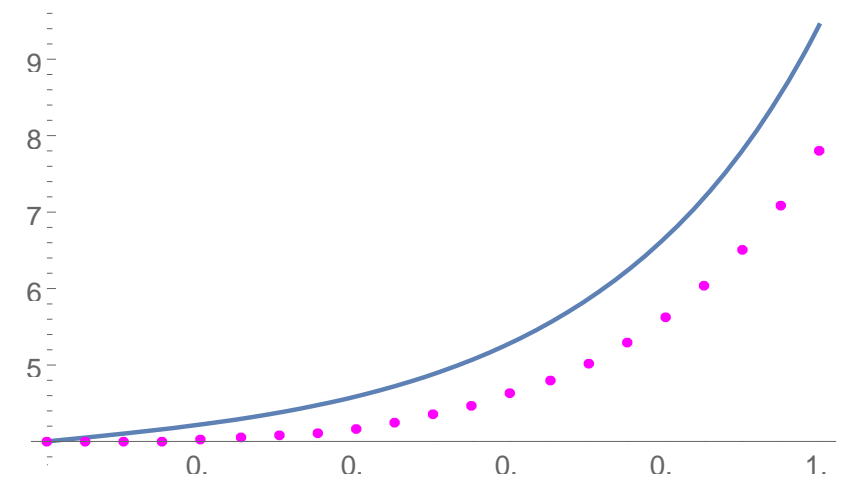

El error absoluto de la esperanza y la varianza de $\mathrm{X}(\mathrm{t})$ con los métodos de Euler y RungeKutta de orden 4 , en $h=\frac{1}{20}$ y $h=\frac{1}{50}$, se representan en los Cuadros 1 y 2 . Se observa que se obtienen mejores aproximaciones con el método de Runge-Kutta. Las Figuras 3 y 4 , representan la comparación de la esperanza y la varianza exacta y aproximada con el método de Runge-Kutta de orden 4 en $h=\frac{1}{20}$. Se observa que la aproximación de la varianza no es buena ya que el método es apropiado para ruidos cuyo comportamiento muestral no es tan irregular como el ruido blanco (no es diferenciable en ningún punto). Para tratar este tipo de incertidumbre se deben aplicar métodos numéricos para ecuaciones diferenciales estocásticas, como el método de Euler-Maruyama 


\subsection{Estudio de un circuito eléctrico con ruido aleatorio}

Consideremos el siguiente circuito RC con parámetros constantes:

$$
\left\{\begin{array}{l}
\mathrm{R} \frac{\mathrm{dQ}(\mathrm{t})}{\mathrm{dt}}+\frac{1}{\mathrm{C}} \mathrm{Q}(\mathrm{t})=\mathrm{V}(\mathrm{t})+\alpha(\mathrm{t}) \mathrm{W}(\mathrm{t}) \\
\mathrm{Q}(0)=\mathrm{Q}_{0}
\end{array}\right.
$$

Donde $Q(t)$ es la carga eléctrica en el tiempo t y $Q_{0}$ es la variable aleatoria exponencial con el parámetro $\lambda=\frac{1}{3}$, independiente de $\mathrm{W}(\mathrm{t})$ para cada $\mathrm{t} \in[0,1]$, lo que significa la carga inicial en el momento $\mathrm{t}=0, \mathrm{y} \mathrm{V}(\mathrm{t})$ son las funciones no aleatorias de la variable tiempo, lo que significa que el voltaje en el tiempo $\mathrm{t} y \mathrm{~W}(\mathrm{t})=\frac{\mathrm{dB}(\mathrm{t})}{\mathrm{dt}}$ es el proceso unidimensional del ruido blanco, $\mathrm{B}(\mathrm{t})$ es el movimiento unidimensional Browniano y $\alpha(\mathrm{t})$ es una función no aleatoria que modeliza la intensidad del ruido en el tiempo $t$.

Ahora, resolviendo la ecuación diferencial estocástica, tenemos

$$
e^{\frac{t}{R C}} d Q(t)+\frac{1}{R C} e^{\frac{t}{R C}} Q(t) d t=\frac{1}{R} e^{\frac{t}{R C}} V(t) d t+\frac{1}{R} \alpha(t) e^{\frac{t}{R C}} d B(t)
$$

Obsérvese que el miembro izquierdo puede escribirse en forma diferencial

$$
d\left(e^{\frac{t}{R C}} Q(t)\right)=\frac{1}{R C} e^{\frac{t}{R C}} Q(t) d t+e^{\frac{t}{R C}} d Q(t)
$$

Por (5.2) y (5.3) tenemos

$\mathrm{Q}(\mathrm{t})=\mathrm{e}^{\frac{-\mathrm{t}}{\mathrm{RC}}}\left[\mathrm{Q}_{0}+\frac{1}{\mathrm{R}} \int_{0}^{\mathrm{t}} \mathrm{e}^{\frac{\mathrm{s}}{\mathrm{RC}}} \mathrm{V}(\mathrm{s}) \mathrm{ds}+\frac{1}{\mathrm{R}} \int_{0}^{\mathrm{t}} \alpha(\mathrm{s}) \mathrm{e}^{\frac{\mathrm{s}}{\mathrm{RC}}} \mathrm{dB}(\mathrm{s})\right]$

Ahora, calculamos $Q_{n}$ por el método aleatorio de Runge-Kutta,

$\mathrm{Q}_{\mathrm{n}+1}=\mathrm{Q}_{\mathrm{n}}+\frac{1}{6}\left(\mathrm{~K}_{1}+2 \mathrm{~K}_{2}+2 \mathrm{~K}_{3}+\mathrm{K}_{4}\right), \mathrm{n}=1,2,3, \ldots$

Donde

$$
\begin{aligned}
& \mathrm{K}_{1}=\frac{\mathrm{h}}{\mathrm{R}}\left[-\frac{1}{\mathrm{C}} \mathrm{Q}_{\mathrm{n}}+\mathrm{V}\left(\mathrm{t}_{\mathrm{n}}\right)+\alpha\left(\mathrm{t}_{\mathrm{n}}\right) \mathrm{W}\left(\mathrm{t}_{\mathrm{n}}\right)\right] \\
& \mathrm{K}_{2}=\frac{\mathrm{h}}{\mathrm{R}}\left[-\frac{1}{\mathrm{C}}\left(1-\frac{\mathrm{h}}{2 \mathrm{RC}}\right) \mathrm{Q}_{\mathrm{n}}-\frac{\mathrm{h}}{2 \mathrm{RC}}\left(\mathrm{V}\left(\mathrm{t}_{\mathrm{n}}\right)+\alpha\left(\mathrm{t}_{\mathrm{n}}\right) \mathrm{W}\left(\mathrm{t}_{\mathrm{n}}\right)\right)+\mathrm{V}\left(\mathrm{t}_{\mathrm{n}}+\frac{\mathrm{h}}{2}\right)\right. \\
& \left.+\alpha\left(t_{n}+\frac{h}{2}\right) w\left(t_{n}+\frac{h}{2}\right)\right] \\
& \mathrm{K}_{3}=\frac{\mathrm{h}}{\mathrm{R}}\left[-\frac{1}{\mathrm{C}}\left(1-\frac{\mathrm{h}}{2 \mathrm{RC}}+\frac{\mathrm{h}^{2}}{4 \mathrm{R}^{2} \mathrm{C}^{2}}\right) \mathrm{Q}_{\mathrm{n}}+\frac{\mathrm{h}^{2}}{4 \mathrm{R}^{2} \mathrm{C}^{2}}\left(\mathrm{~V}\left(\mathrm{t}_{\mathrm{n}}\right)+\alpha\left(\mathrm{t}_{\mathrm{n}}\right) \mathrm{W}\left(\mathrm{t}_{\mathrm{n}}\right)\right)+\left(1-\frac{\mathrm{h}}{2 \mathrm{RC}}\right)\left(\mathrm{V}\left(\mathrm{t}_{\mathrm{n}}+\frac{\mathrm{h}}{2}\right)+\alpha\left(\mathrm{t}_{\mathrm{n}}+\right.\right.\right. \\
& \left.\left.\left.\frac{\mathrm{h}}{2}\right) \mathrm{~W}\left(\mathrm{t}_{\mathrm{n}}+\frac{\mathrm{h}}{2}\right)\right)\right] \\
& \mathrm{K}_{4}=\frac{\mathrm{h}}{\mathrm{R}}\left[-\frac{1}{\mathrm{C}}\left(1-\frac{\mathrm{h}}{\mathrm{RC}}+\frac{\mathrm{h}^{2}}{2 \mathrm{R}^{2} \mathrm{C}^{2}}-\frac{\mathrm{h}^{3}}{4 \mathrm{R}^{3} \mathrm{C}^{3}}\right) \mathrm{Q}_{\mathrm{n}}-\frac{\mathrm{h}^{3}}{4 \mathrm{R}^{3} \mathrm{C}^{3}}\left(\mathrm{~V}\left(\mathrm{t}_{\mathrm{n}}\right)+\alpha\left(\mathrm{t}_{\mathrm{n}}\right) \mathrm{W}\left(\mathrm{t}_{\mathrm{n}}\right)\right)-\frac{\mathrm{h}}{\mathrm{RC}}\left(1-\frac{\mathrm{h}}{2 \mathrm{RC}}\right)\left(\mathrm{V}\left(\mathrm{t}_{\mathrm{n}}+\frac{\mathrm{h}}{2}\right)+\right.\right. \\
& \left.\left.\alpha\left(t_{n}+\frac{h}{2}\right) w\left(t_{n}+\frac{h}{2}\right)\right)+V\left(t_{n}+h\right)+\alpha\left(t_{n}+h\right) W\left(t_{n}+h\right)\right] \\
& \mathrm{Q}_{\mathrm{n}+1}=\mathrm{aQ}_{\mathrm{n}}+\mathrm{b}_{\mathrm{n}}, \mathrm{n}=1,2,3, \ldots \\
& \mathrm{Q}_{\mathrm{n}}=\mathrm{a}^{\mathrm{n}} \mathrm{Q}_{0}+\sum_{\mathrm{i}=0}^{\mathrm{n}-1} \mathrm{a}^{\mathrm{n}-\mathrm{i}-1} \mathrm{~b}_{\mathrm{i}}, \mathrm{n}=1,2,3, \ldots
\end{aligned}
$$


De (5.4) y (5.6), obtenemos las esperanza y las varianzas de $Q_{t}$ y $Q_{n}$.

$\mathbb{E}[\mathrm{Q}(\mathrm{t})]=\mathrm{e}^{\frac{-\mathrm{t}}{\mathrm{RC}}}\left[3+\frac{1}{\mathrm{R}} \int_{0}^{\mathrm{t}} \mathrm{e}^{\frac{\mathrm{s}}{\mathrm{RC}}} \mathrm{V}(\mathrm{s}) \mathrm{ds}\right]$

$\mathbb{E}[Q(t)]=3 a^{n}+\sum_{i=0}^{n-1} a^{n-i-1}\left(\frac{h}{6 R}\left[1-\frac{h}{R C}+\frac{h^{2}}{2 R^{2} C^{2}}-\frac{h^{3}}{4 R^{3} C^{3}}\right] V\left(t_{i}\right)+\frac{h}{3 R}[1+(1-\right.$

$\left.\left.\left.\frac{\mathrm{h}}{2 \mathrm{RC}}\right)^{2}\right] \mathrm{V}\left(\mathrm{t}_{\mathrm{i}}+\frac{\mathrm{h}}{2}\right)+\frac{\mathrm{h}}{6 \mathrm{R}} \mathrm{V}\left(\mathrm{t}_{\mathrm{i}}+\mathrm{h}\right)\right)$

$\mathrm{Y} \quad \operatorname{Var}[\mathrm{Q}(\mathrm{t})]=\exp \left(\frac{-2 \mathrm{t}}{\mathrm{RC}}\right)\left[9+\frac{1}{\mathrm{R}^{2}} \int_{0}^{\mathrm{t}} \alpha^{2}(\mathrm{~s}) \exp \left(\frac{2 \mathrm{~s}}{\mathrm{RC}}\right) \mathrm{ds}\right]$

Donde $\quad A_{i, k}=\frac{h^{2}}{36 R^{2}}\left[1-\frac{h}{R C}+\frac{h^{2}}{2 R^{2} C^{2}}-\frac{h^{3}}{4 R^{3} C^{3}}\right]^{2} \alpha\left(t_{i}\right) \alpha\left(t_{k}\right)+\frac{h^{2}}{9 R^{2}}\left[1+\left(1-\frac{h}{2 R C}\right)^{2}\right]^{2} \times \alpha\left(t_{i}+\right.$ $\left.\frac{h}{2}\right) \alpha\left(t_{k}+\frac{h}{2}\right)+\frac{h^{2}}{36 R^{2}} \alpha\left(t_{i}+h\right) \alpha\left(t_{k}+h\right)$

$$
\begin{gathered}
\mathrm{B}_{\mathrm{i}, \mathrm{k}}=\frac{\mathrm{h}^{2}}{18 \mathrm{R}^{2}}\left[1-\frac{\mathrm{h}}{\mathrm{RC}}+\frac{\mathrm{h}^{2}}{2 \mathrm{R}^{2} \mathrm{C}^{2}}-\frac{\mathrm{h}^{3}}{4 \mathrm{R}^{3} \mathrm{C}^{3}}\right]\left[1+\left(1-\frac{\mathrm{h}}{2 \mathrm{RC}}\right)^{2}\right] \alpha\left(\mathrm{t}_{\mathrm{i}}\right) \alpha\left(\mathrm{t}_{\mathrm{k}}+\frac{\mathrm{h}}{2}\right) \\
+\frac{\mathrm{h}^{2}}{18 \mathrm{R}^{2}}\left[1+\left(1-\frac{\mathrm{h}}{2 \mathrm{RC}}\right)^{2}\right] \alpha\left(\mathrm{t}_{\mathrm{i}}+\frac{\mathrm{h}}{2}\right) \alpha\left(\mathrm{t}_{\mathrm{k}}+\mathrm{h}\right) \\
\mathrm{C}_{\mathrm{i}, \mathrm{k}}=\frac{\mathrm{h}^{2}}{36 \mathrm{R}^{2}}\left[1-\frac{\mathrm{h}}{\mathrm{RC}}+\frac{\mathrm{h}^{2}}{2 \mathrm{R}^{2} \mathrm{C}^{2}}-\frac{\mathrm{h}^{3}}{4 \mathrm{R}^{3} \mathrm{C}^{3}}\right] \alpha\left(\mathrm{t}_{\mathrm{i}}\right) \alpha\left(\mathrm{t}_{\mathrm{k}}+\mathrm{h}\right), \quad \mathrm{i}, \mathrm{k}=0,1,2, \ldots, \mathrm{n}-1
\end{gathered}
$$

El error absoluto de la esperanza y la varianza de $Q_{n} \operatorname{con} V(t)=\exp (t), \alpha(t)=\frac{\sin (t)}{25}, R=1$ y $\mathrm{C}=2$ está representada en el Cuadro 5.1.

El error absoluto de la esperanza y varianza de $Q_{n} \operatorname{con} V(t)=\exp (t), \alpha(\mathrm{t})=\frac{\sin (\mathrm{t})}{25}, \mathrm{R}=1 \mathrm{y}$ $\mathrm{C}=2$ están representadas en la Figura 5.3 y 5.6.

\section{Cuadro 3}

Error absoluto de la esperanza y la varianza de $Q_{n}$ con $h=\frac{1}{20}$ del estudio de un circuito eléctrico con ruido aleatorio 5 .

\begin{tabular}{ccc}
\hline t & Esperanza & Varianza \\
\hline 0.1 & $1,8186 \times 10^{-9}$ & $7,4434 \times 10^{-8}$ \\
0.2 & $3,6829 \times 10^{-9}$ & $1,1779 \times 10^{-6}$ \\
0.3 & $5,6077 \times 10^{-9}$ & $5,6969 \times 10^{-6}$ \\
0.4 & $7,6086 \times 10^{-9}$ & $1,7054 \times 10^{-5}$ \\
0.5 & $9,7022 \times 10^{-9}$ & $3,9168 \times 10^{-5}$ \\
0.6 & $1,1906 \times 10^{-8}$ & $7,5901 \times 10^{-5}$ \\
0.7 & $1,4240 \times 10^{-8}$ & $1,3052 \times 10^{-4}$ \\
0.8 & $1,6725 \times 10^{-8}$ & $2,0527 \times 10^{-4}$ \\
0.9 & $1,9383 \times 10^{-8}$ & $3,0096 \times 10^{-4}$ \\
1.0 & $2,2239 \times 10^{-8}$ & $4,1676 \times 10^{-4}$ \\
\hline
\end{tabular}

Fuente: El Autor 


\section{Figura 5}

Esperanza $X(t)$ mediante el método Runge-Kutta de orden 4 y $h=\frac{1}{20}$ del estudio de un circuito eléctrico con ruido aleatorio 5.

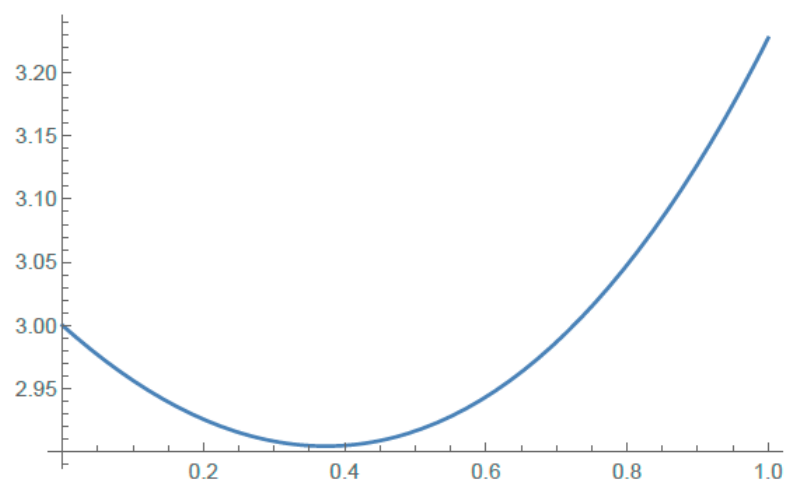

Fuente: El Autor

Figura 6

Varianza $X(t)$ y $X_{n}$ mediante el método Runge-Kutta de orden 4 y $h=\frac{1}{20}$, del estudio de un circuito eléctrico con ruido aleatorio 5

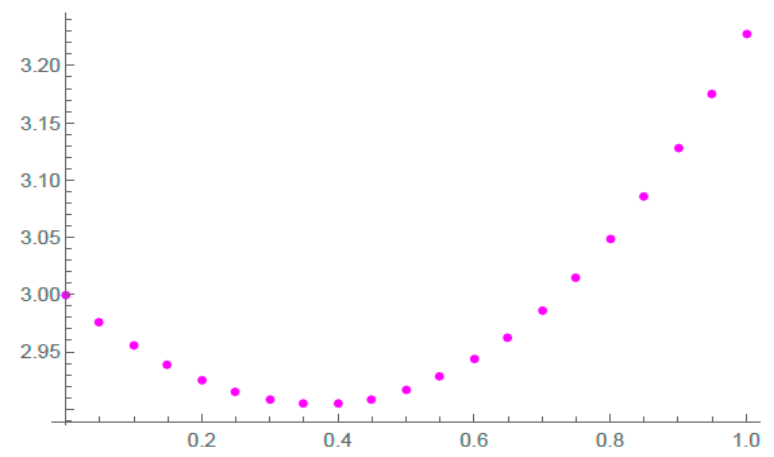

Fuente: El Autor

\section{Conclusiones.}

- Dentro del estudio de la aproximación numérica de ecuaciones diferenciales con el proceso de aleatoriedad, el estudio de la convergencia y sus aplicaciones, se ha planteado en primer lugar el esquema aleatorio de Euler que comprende el caso escalar y matricial demostrando el comportamiento de algunas condiciones en las cuales la convergencia referente a la media cuadrática se presenta estable y calcula la esperanza y la varianza exacta aproximada.

- El esquema Euler permite llegar a la conclusión de que la convergencia lenta y el aspecto de restricción de su región de estabilidad absoluta nos hace considerar otros métodos donde la convergencia es mayor por lo cual se plantea el estudio adicional del esquema aleatorio Runge- Kutta, siendo un método superior al de Euler por lo cual su orden de convergencia global es cuarto.

- El método de Runge Kutta y Euler permite determinar y analizar varios casos donde se aprecian tipos de ruidos con el comportamiento muestral regular. en el caso 
específico de ruidos blanco que son característicos y muy irregulares al no ser diferenciales en ningún punto conducen a otro tipo de ecuaciones diferenciales con cierto grado de incertidumbre denominadas ecuaciones diferenciales estocásticas cuya aproximación se aborda con técnicas alternativas como el método de EulerMaruyama que es considerado de orden superior.

- Concluyendo que el método de Runge Kutta de cuarto orden representa la mejor elección al momento de proporcionar y trabajar con pequeños márgenes de errores con respecto a la solución real del problema resaltando la ventaja de que es fácilmente programable en un software para realizar las diferentes interacciones necesarias, obteniendo resultados en cuanto a la esperanza y varianza de tipo senoidal, la cual ha sido producida por la generación de la corriente alterna.

\section{Referencias bibliográficas.}

Aldana, S., Vereda, F., Hidalgo-Alvarez, R., \& de Vicente, J. (2016). Facile synthesis of magnetic microfibers by directed selfassembly. Polymer, 93, 61-64.

Azor, Ainhoa, Juan Carlos Cort, Dolores Rosell, Ster E. N. Investigaci, and Tica Septiembre. 2020. "Solución Numérica de Ecuaciones Diferenciales Con Incertidumbre y Aplicaciones."

Azorín Penalva, A. (2020). Solución numérica de ecuaciones diferenciales con incertidumbre y aplicaciones.

Bossis, G., Marins, J., Kuzhir, P., Volkova, O., \& Zubarev, A. (2015). Functionalized

Cortés, J., Jódar L., y Villafuerte L., (2007). Numerical solution of random differential equations: a mean square approach. Math Comput. Model. 45, 757-765.

Henrici, P., (1962). Discrete Variable Methods in Ordinary Differential Equations. John Wiley and Sons, New York.

Lecca, E. R., \& Puente, M. M. (2006). Aplicaciones computacionales de las ecuaciones diferenciales estocásticas. Industrial Data, 9(1), 64-75.

Oksendal, B., (1998). Stochastic Differential Equations: An Introduction with Applications. Springer, New York, 5th edn.

Khodabin M y Rostami M., (2015). Mean square numerical solution of stochastic differential equations by fourth order Runge-Kutta method and its application in the electric circuits with noise. Advances in Difference Equations, 62, 1-19. [2] L.

Villafuerte A (2007). Numerical and Analytical Mean Square Solutions for Random Differential Models. Tesis Doctoral, Universidad Politécnica de Valencia

Seminario, Ricardo. 2012. "Metodos Númericos Para Ingenieria.” Libro 69. 
Soong, T., (1973). Random Diferential Equations in Science and Engeneering. Academic Press, New York.

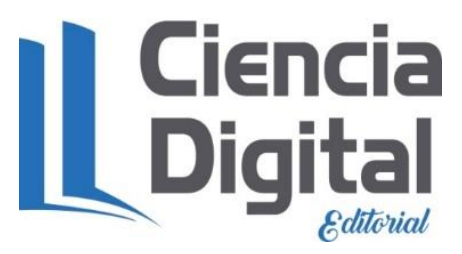




\section{PARA CITAR EL ARTÍCULO INDEXADO.}

Penalva Ainhoa, A., \& Yaulema Castañeda, J. L. (2021). Análisis del caso Solución numérica de ecuaciones diferenciales con incertidumbre y aplicaciones. ConcienciaDigital, $\quad 4(3.1)$, 253-272. https://doi.org/10.33262/concienciadigital.v4i3.1.1828

\section{Ciencia
Digital
$\varepsilon$ thatolal}

El artículo que se publica es de exclusiva responsabilidad de los autores y no necesariamente reflejan el pensamiento de la Revista Conciencia Digital.

El artículo queda en propiedad de la revista y, por tanto, su publicación parcial y/o total en otro medio tiene que ser autorizado por el director de la Revista Conciencia Digital.

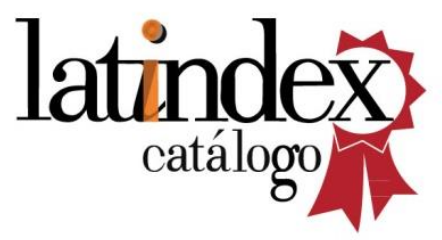

